\title{
Téoros
}

Revue de recherche en tourisme

\section{Tourism and the Consumption of Wildlife - Hunting, Shooting and Sport Fishing, Brent LOVELOCK (sous la direction de) Londres, Angleterre : Routledge, 2008, 290 pages \\ ISBN-10 : 0-415-40381-2}

\section{Alain A. Grenier}

Volume 32, numéro 1, 2013

URI : https://id.erudit.org/iderudit/1036728ar

DOI : https://doi.org/10.7202/1036728ar

Aller au sommaire du numéro

Éditeur(s)

Université du Québec à Montréal

ISSN

0712-8657 (imprimé)

1923-2705 (numérique)

Découvrir la revue

Citer cette note

Grenier, A. A. (2013). Tourism and the Consumption of Wildlife - Hunting, Shooting and Sport Fishing, Brent LOVELOCK (sous la direction de) Londres, Angleterre : Routledge, 2008, 290 pages ISBN-10 : 0-415-40381-2. Téoros, 32(1), 143-144. https://doi.org/10.7202/1036728ar d'utilisation que vous pouvez consulter en ligne. 


\section{POUR EN LIRE PLUS}

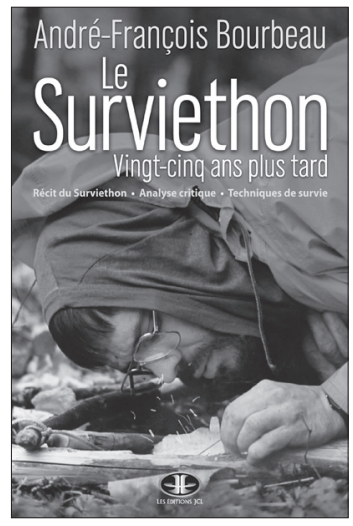

Le Surviethon : vingt-cinq ans plus tard

André-François BOURBEAU

Chicoutimi, Canada : Les éditions JCL, 2011, 512 pages

ISBN : 978-2-89431-434-0

En 1988, André-François Bourbeau publiait Surviethon, au gré de la nature, le récit quotidien de l'expérience scientifique et humaine qui l'avait conduit, avec son compagnon d'aventure Jacques Montminy, à passer trente et un jours en forêt sans équipement et en autonomie complète, établissant ainsi un record Guinness de survie volontaire en milieu sauvage.

Fin 2011, la sortie du Surviethon : vingt-cinq ans plus tard est bien plus qu'une réédition. Si le récit initial se retrouve in extenso dans le nouvel ouvrage, celui-ci est unique à de nombreux égards.

La force du temps a donné à l'auteur, désormais professeur émérite à l'Université du Québec à Chicoutimi, le recul nécessaire pour porter un regard critique sur les décisions prises à l'époque. Cette rétroanalyse est d'autant plus pertinente qu'elle se fonde sur le désir manifeste d'expliquer sans compromis ces choix et de proposer des options davantage éprouvées. En effet, depuis l'expérience initiale, l'auteur n'a cessé de développer ses compétences et le présent livre traduit bien cette longue acquisition de connaissances.

L'ouvrage traite de la survie en forêt selon une approche radicalement différente des écrits traditionnels en la matière. Au cours de sa carrière comme professeur et chercheur, André-François Bourbeau s'est efforcé d'évaluer scientifiquement les techniques, les outils et les méthodes de survie afin de valider leur pertinence respective.

Au cours des dernières années, l'équipe du Laboratoire d'expertise et de recherche en plein air (LERPA), sous la direction du professeur Bourbeau, a conçu deux outils novateurs dont il est abondamment question dans ce livre. Le premier est un protocole de prise de décision en cas d'urgence en forêt appelé le modèle SÉRA (secours-énergie-risques-atouts). Unique en son genre, cet outil directement utilisable regroupe les principes théoriques de gestion des risques et de réaction aux situations de survie. Le second, le modèle AEPS, est un outil d'auto-évaluation du potentiel de survie qui a pour but de permettre à un individu d'estimer sa capacité à gérer une situation dans laquelle sa vie est mise en danger, en fonction de quatre facteurs : le savoir-faire, l'état psychologique, les capacités physiologiques et la capacité d'analyse décisionnelle.

Bien qu'empreint d'une volonté de suivre une démarche scientifique, l'auteur ne verse pas pour autant dans un hermétique langage réservé aux experts. L'ouvrage demeure très accessible, schémas et photos agrémentant le tout. Notons qu'outre le récit de 1988 et l'analyse méthodique des décisions prises et des stratégies privilégiées alors, le livre présente une compilation de techniques inédites visant l'accroissement de l'autonomie en milieu naturel. Il lance également un cri d'alarme fort à propos et invite à une protection accrue et systématique de la faune et de la flore.

L'ouvrage Le Surviethon : vingt-cinq ans plus tard est appelé à devenir une référence dans le domaine, en raison de l'expertise de son auteur, de la pertinence et de l'aspect novateur des outils et des approches scientifiques utilisés, et de la somme des connaissances qui le parsèment, résultat d'une vie de praticien.

Manu TRANQUARD, Ph.D., LL.M., Professeur au Département des sciences humaines,
Chercheur au Laboratoire d'expertise et de recherche en plein air (LERPA),
Université du Québec à Chicoutimi (UQAC)

Tourism and the Consumption of Wildlife - Hunting, Shooting and Sport Fishing Brent LOVELOCK (sous la direction de)

Londres, Angleterre : Routledge, 2008, 290 pages

ISBN-10 : 0-415-40381-2

Comme l'indique son titre, cet ouvrage explore la question encore peu étudiée du tourisme de chasse et de pêche, un type de tourisme plus controversé que tout autre (exception faite du tourisme sexuel impliquant des mineurs). 


\section{POUR EN LIRE PLUS}

L'ouvrage regroupe, sous forme de chapitres, une collection de vingt textes traitant du concept et de l'historique de la chasse, de la combinaison de celle-ci au tourisme, des impacts de ce type de tourisme dit «de consommation» (consumptive wildlife tourism) et, enfin, des enjeux actuels liés à son développement. On y présente le niveau de complexité du sujet, la diversité des expériences recherchées par les touristes (la connexion avec les aliments, le contact avec la nature, les pratiques de cueillette traditionnelles ou technologiques), ainsi que les problèmes de gestion (conflits entre chasseurs ou avec des résidents chasseurs et non chasseurs, établissement de politiques, etc.).

Le livre éveille également le lecteur aux situations particulièrement problématiques des usages multiples du territoire, comme dans le cas de la pêche professionnelle ou de l'exploitation industrielle d'un lieu à des fins énergétiques. Les impacts du jumelage des activités de cueillette au tourisme sont nombreux et pas toujours positifs. L'ouvrage compare enfin les retombées économiques de la chasse à celles du simple tourisme d'observation de la faune et démontre comment les activités de chasse et de pêche peuvent aider les communautés à valoriser leur patrimoine naturel.

Si les exemples tirés aux quatre coins du monde offrent un éventail intéressant de la situation du tourisme de chasse et de pêche dans des contextes géoculturels relativement divers (du Nunavut à la Nouvelle-Zélande, en passant par l'Afrique), on reprochera néanmoins à l'éditeur de s'en être tenu au milieu anglo-saxon. De même, on regrettera que l'ouvrage ne se penche pas, à l'instar de notre dossier thématique, sur la notion du droit des animaux, autrement qu'à titre d'obstacle au développement des pratiques de chasse. Cette question, encore trop facilement évacuée, devra un jour ou l'autre être étudiée en profondeur. Dans la réalité, les positions mises de l'avant ne peuvent pas être toujours aussi extrêmes que la littérature nous le laisse croire.

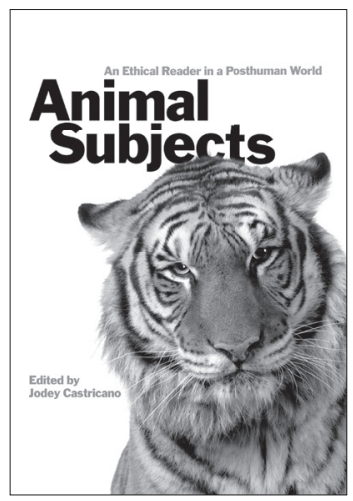

\section{Animal Subjects - An Ethical Reader in a Posthuman World}

Jodey CASTRICANO (sous la direction de)

Waterloo, Canada : Wilfrid Laurier University Press, 2008, 312 pages

ISBN-13 : 978-0-88920-512-3

L'éthique en matière de droits des animaux a longtemps été la «chasse gardée » de penseurs comme Tom Regan et Peter Singer. Bravant toutes les polémiques, leur courant de pensée a fait école et voilà qu'une nouvelle génération d'universitaires prend la relève. Sous la direction de Jodey Castricano de l'Université de la Colombie-Britannique en Okanagan, cet ouvrage réunit les travaux de treize chercheurs et chercheuses, qui posent un regard tout aussi critique sur le rapport entre les humains et le reste du monde animal.

Castricano et ses collègues s'attardent à la problématique entourant le débat sur la question du respect des animaux dans les pratiques et les études culturelles. Leur point de départ : une critique de la structure du monde actuel, dont ils dénoncent l'aspect hiérarchique d'un système organisé autour du genre, de l'ethnicité, du sexe et des classes sociales. Or, les études culturelles qui s'emploient notamment à démontrer ces constructions ont, selon les auteurs, exclu le monde animal. L'ouvrage vise donc à rectifier cet «oubli». (suite en page 147)

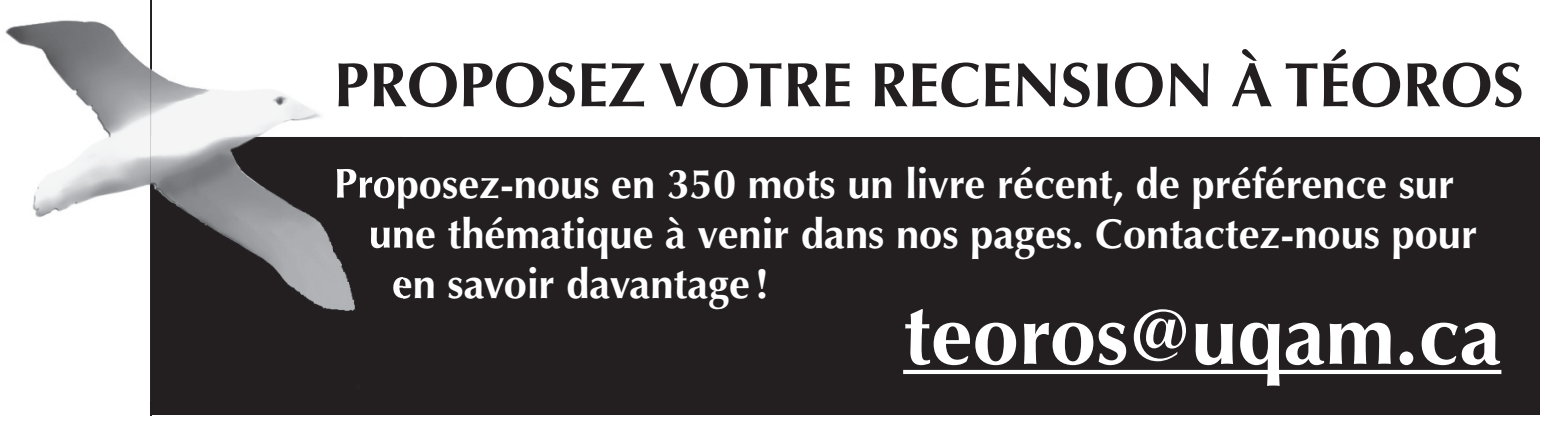

